\title{
A NOTE ON WEIGHTED NORM INEQUALITIES FOR THE HARDY-LITTLEWOOD MAXIMAL OPERATOR
}

\author{
MICHAEL CHRIST AND ROBERT FEFFERMAN
}

\begin{abstract}
In this note we give an extremely simple proof of the weight norm inequalities for the Hardy-Littlewood maximal operator in $\mathbf{R}^{n}$
\end{abstract}

The purpose of this note is to provide an extremely elementary proof of the boundedness of the maximal operator, $\|M f\|_{L^{r^{(}\left(w^{\prime}\right)}} \leqslant C_{p}\|f\|_{L^{p^{\prime}\left(w^{\prime}\right)}}$ when the weight $w \in A_{p}, p>1$. The result is well known and is due to Muckenhoupt [1]. The proof was simplified in R. Coifman and C. Fefferman [2], but still depended on a "reverse Hölder" inequality and was not totally elementary. Rather recently Sawyer in [3] solved the two weight problem for the maximal operator, which yielded a simple new proof in the one weight case, but which involved a condition on the weight not obviously equivalent to $A_{p}$. In [4] Hunt, Kurtz, and Neugebauer proved this equivalence and their proof avoids the reverse Hölder inequality. Finally, our proof avoids both the reverse Hölder inequality and also the reformulation of the $A_{p}$ condition. The proof is very similar to Sawyer's [3] and Christ's alternate proof to Sawyer's [5].

We shall assume the reader is familiar with the basic definitions from weighted inequalities as appear in, say, [2]. Then, let $f \in L^{p}(w)$ where $w \in A_{p}\left(\mathbf{R}^{n}\right), p>1$. We Calderón-Zygmund decompose $f$ at heights $C_{n}^{k}$ for $k$ an integer, where $C_{n}$ is some large constant depending only on the dimension $n$, and which we specify later. Let $Q_{j}^{k}, j \geqslant 1$, be the Calderón-Zygmund cubes at height $C_{n}^{k}$. Let $E_{j}^{k}=Q_{j}^{k}-$ $\cup_{Q_{m}^{\prime} \subset Q^{k}} Q_{m}^{\prime}$. Then we have

$$
\int_{\mathbf{R}^{n}}(M f)^{p} w \leqslant B \sum_{k, j}\left(\frac{1}{\left|Q_{j}^{k}\right|} \int_{Q_{,}^{k}} f\right)^{p} w\left(E_{j}^{k}\right)
$$

(henceforth we write $q(E)$ for $\int_{E} q$ where $q$ is in $L_{\text {loc }}^{1}\left(\mathbf{R}^{n}\right), q_{E}=q(E) /|E|$, and following Sawyer, set $\left.\sigma=w^{-1 /(p-1)}\right)$

$$
\leqslant B \sum_{k, j}\left[\frac{1}{\sigma\left(Q_{j}^{k}\right)} \int_{Q_{j}^{k}}\left(f \sigma^{-1}\right) \sigma\right]^{p} \sigma\left(E_{j}^{k}\right) \cdot\left\{\frac{w\left(E_{j}^{k}\right)}{\sigma\left(E_{j}^{k}\right)}\left(\frac{\sigma\left(Q_{j}^{k}\right)}{\left|Q_{j}^{k}\right|}\right)^{p}\right\} .
$$

Received by the editors June 15, 1982.

1980 Mathematics Subject Classification. Primary 42B25.

Key' words and phrases. Maximal functions, weights. 
So far we have just used arithmetic. Now observe that if $C_{n}$ is large enough $\left|E_{j}^{k}\right|>$ $\frac{1}{2}\left|Q_{j}^{k}\right|$. Since $\sigma \in A_{p^{\prime}}$, it is $A_{\infty}$ and so $\sigma\left(E_{j}^{k}\right) \geqslant \eta\left(\sigma\left(Q_{j}^{k}\right)\right)$ for some $\eta>0$. (Indeed, assuming $\sigma_{Q_{1}^{k}}=1$, then $\sigma\left(E_{j}^{k}\right) / \sigma\left(Q_{j}^{k}\right) \geqslant \frac{1}{2} \sigma_{E_{j}^{k}}$. But

$$
\left.\left(\sigma_{E_{l}^{k}}\right)^{-1 /\left(p^{\prime}-1\right)} \leqslant\left(\sigma^{-1 /\left(p^{\prime}-1\right)}\right)_{E_{l}^{\alpha}} \leqslant 2\|\sigma\|_{A_{p^{\prime}}}^{1 /\left(p^{\prime}-1\right)} .\right)
$$

Taking this into account, the quantity in braces is bounded since $w \in A_{p}$. What is left is just dominated by

$$
\int_{\mathbf{R}^{n}} M_{\boldsymbol{\sigma}}^{p}\left(f \boldsymbol{\sigma}^{-1}\right) \boldsymbol{\sigma}
$$

(where $M_{\mu}(f)(x)=\sup _{x \in Q}(1 / \mu(Q)) \int_{Q}|f| d \mu$ for a measure $\mu$ ). But $M_{\sigma}$ is bounded on $L^{p}(\sigma)$ and so $(*)$

$$
\leqslant B^{\prime} \int_{\mathbf{R}^{n}} f^{p} \boldsymbol{\sigma}^{-p+1}=B^{\prime} \int_{\mathbf{R}^{n}} f^{p} w
$$

\section{BIBLIOGRAPHY}

1. B. Muckenhoupt, Weighted norm inequalities for the Hardl maximal function. Trans. Amer. Math. Soc. 165 (1972), 207-226.

2. R. Coifman and C. Fefferman, Weighted norm inequalities for maximal functions and singular integrals, Studia Math. 51 (1974). 241-250.

3. E. Sawyer, A characterization of a two neight norm inequality for maximal operators (to appear).

4. R. Hunt. D. Kurtz and C. Neugebauer, $A$ note on the equivalence of $A_{p}$ and San'yer's condition for equal neights (to appear).

5. M. Christ, Weighted norm inequalities and Schur's lemma (preprint).

Department of Mathematics, University of Chicago, Chicago, Illinois 60637 (Current address of Robert Fefferman)

Current address (Michael Christ): Department of Mathematics, Princeton University, Princeton. New Jersey 08544 